\title{
Fractional analysis of bronchoalveolar lavage in systemic sclerosis-associated interstitial lung disease
}

\author{
Kazumasa Kase $^{1,2}$, Satoshi Watanabe ${ }^{1 \wedge}$, Keigo Saeki ${ }^{1}$, Yuko Waseda ${ }^{3}$, Hazuki Takato ${ }^{2}$, Yukari Ichikawa ${ }^{4}$, \\ Akari Murata $^{5}$, Masahide Yasui ${ }^{6}$, Ohkura Noriyuki ${ }^{1}$, Johsuke Hara ${ }^{1}$, Takashi Sone ${ }^{1}$, Miki Abo ${ }^{1}$, \\ Hideharu Kimura ${ }^{1}$, Kazuo Kasahara ${ }^{1}$
}

${ }^{1}$ Respiratory Medicine, Kanazawa University Graduate School of Medical Sciences, Kanazawa, Ishikawa, Japan; ${ }^{2}$ Respiratory Medicine, Japan Community Health Care Organization, Kanazawa Hospital, Kanazawa, Ishikawa, Japan; ${ }^{3}$ Third Department of Internal Medicine, Faculty of Medical Sciences, University of Fukui, Fukui, Japan; ${ }^{4}$ Respiratory Medicine, Kanazawa Municipal Hospital, Kanazawa, Ishikawa, Japan; ${ }^{5}$ Respiratory Medicine, Keiju Medical Center, Nanao, Ishikawa Japan; ${ }^{6}$ Respiratory Medicine, National Hospital Organization Nanao Hospital, Nanao, Ishikawa, Japan

Contributions: (I) Conception and design: All authors; (II) Administrative support: K Kase, S Watanabe, K Saeki; (III) Provision of study materials or patients: All authors; (IV) Collection and assembly of data: All authors; (V) Data analysis and interpretation: All authors; (VI) Manuscript writing: All authors; (VII) Final approval of manuscript: All authors.

Correspondence to: Satoshi Watanabe, MD, PhD. Respiratory Medicine, Kanazawa University Graduate School of Medical Sciences, 13-1 Takaramachi, Kanazawa Ishikawa, 920-8641, Japan. Email: swatanabe1114152@gmail.com.

Background: The utility of bronchoalveolar lavage (BAL) in the evaluation of systemic sclerosis-associated interstitial lung disease (SSc-ILD) remains controversial. Fractional analysis of BAL (FBAL) is a technique that can analyze small airways and alveolar compartments separately and has proven informative in other ILDs. The aim of this study was to explore FBAL characteristics across the spectrum of SSc-ILD severity.

Methods: We retrospectively reviewed patients with SSc-ILD who underwent bronchoscopy with FBAL using three $50 \mathrm{~mL}$ aliquots of saline solution. These aliquots were analyzed separately for differential cell composition (FBAL-1, -2, and -3). We compared the FBAL cell composition to the progression of ILD and end-stages of ILD using Cox proportional hazards models.

Results: Sixty-eight patients with SSc-ILD were enrolled in this study. The percentage of neutrophils and eosinophils was lower in FBAL-3 compared to FBAL-1. In contrast, the percentage of macrophages and lymphocytes was higher in FBAL-3. Neutrophils in FBAL-2, -3, and the estimated total FBAL cell fraction (FBAL-total) were negatively correlated with the forced vital capacity \% predicted ( $\mathrm{r}=-0.420,-0.362$, -0.409 , respectively). Although FBAL-total was not linked to the progression and end-stage of ILD, a high percentage of neutrophils in FBAL-3 was significantly associated with the development of end-stage ILD (HR 1.093, 95\% CI: 1.003-1.190).

Conclusions: A higher percentage of neutrophils in FBAL-3 is correlated with development of end-stage ILD in SSc-ILD as well as mortality.

Keywords: Fractional bronchoalveolar lavage; systemic sclerosis (SSc); scleroderma; interstitial lung disease (ILD)

Submitted Aug 04, 2020. Accepted for publication May 20, 2021.

doi: $10.21037 /$ jtd-20-2596

View this article at: https://dx.doi.org/10.21037/jtd-20-2596

\footnotetext{
$\wedge$ ORCID: 0000-0002-2579-8472.
} 


\section{Introduction}

Interstitial lung disease (ILD) is a life-threatening complication of systemic sclerosis (SSc) that is present in over $80 \%$ of SSc patients (1). Although most patients with SSc-ILD have a stable or slowly progressive form of the disease, $25-30 \%$ will ultimately progress to respiratory failure or death (2). Pulmonary function tests including a forced vital capacity (FVC) and diffusing capacity of lung for carbon monoxide (DLco) are often used to estimate the severity of ILD (3-6). However, FVC and DLco alone are poor predictors of SSc-ILD prognosis $(7,8)$. New therapies such as novel immunosuppressive regimens, antifibrotics, and hematopoietic stem cell transplantation are extremely promising (9-11), but decisions regarding the timing of initiation and optimal patient selection remain unclear (12). Elucidating predictors of disease progression and prognosis is important to determine appropriate therapy and timing for patients with SSc-ILD.

Bronchoalveolar lavage (BAL) is a common and welltolerated procedure for the diagnosis and evaluation of patients with various lung diseases including infections, malignancies, and ILDs. The lavage fluid can be assessed with a variety of analytical tests, including differential cell counts, cytopathologic analyses, and cultures, in addition to specific molecular and immunologic diagnostic tests. While differential cell count profiles in the BAL fluid may not be specific or diagnostic, they are helpful for managing challenging cases and improving diagnostic accuracy (13). For example, although the cellular analysis of BAL fluid was not recommended for patients clinically suspected of having idiopathic pulmonary fibrosis (IPF) (14), a recent guideline suggested that a BAL fluid cellular lymphocyte analysis might play a key role in distinguishing fibrotic HP from IPF (15). Recent studies have shown new scores for discriminating between healthy and diseased individuals (16) and differentiating common variable immunodeficiencyassociated ILD from sarcoidosis (17).

In contrast, the utility of BAL in the assessment of SSc-ILD has been controversial; some have found that an abnormal BAL cellular profile, especially neutrophilia or eosinophilia, is associated with greater severity of ILD (18-22), while others have found no such association (23-25). Thus, the current use of BAL cellular analysis for SSc lung disease is restricted to the investigation of different types of infections (26).

Fractional BAL (FBAL) is a technique that analyzes smaller, serially collected BAL aliquots independently. Fraction 1 (FBAL-1) predominantly samples the milieu at the level of peripheral airways while fractions 2 and 3 (FBAL-2, -3) sample the alveolar compartment (27). FBAL is able to resolve inflammatory cell populations more precisely and better reflect the condition of several diseases including cystic fibrosis, hypersensitivity pneumonitis, and sarcoidosis $(27,28)$. However, the characteristics of FBAL in SSc-ILD has not been previously investigated.

We hypothesized that FBAL would more accurately predict outcomes of SSc-ILD compared to pooled BAL. This study aimed to evaluate the prognostic value of FBAL in patients with SSc-ILD.

We present the following article in accordance with the STROBE reporting checklist (available at https://dx.doi. org/10.21037/jtd-20-2596).

\section{Methods}

\section{Patients}

A retrospective cohort study was conducted on the consecutive patients with SSc-ILD presenting to the Department of Respiratory Medicine, Kanazawa University Hospital who underwent FBAL from January 2005 to December 2017. We enrolled adult patients who were diagnosed with SSc by a dermatologist or rheumatologist using the classification criteria of the American College of Rheumatology and European League Against Rheumatism (29). The presence of ILD was confirmed using chest high-resolution computed tomography (HRCT) scan by pulmonologists and radiologists. The ILD pattern on HRCT scan was classified according to the official American Thoracic Society/European Respiratory Society (ATS/ERS) statement (14). Clinical data including age, gender, smoking status, steroid or immunosuppressant use, the classification of SSc, the modified Rodnan skin score (mRSS), autoantibodies, chest HRCT findings, and pulmonary function tests at the time of the BAL, and serial pulmonary function tests data, were collected. We assessed the prognostic value for the patients who have an FVC of at least $45 \%$ of the predicted value and a DLco ranging from $30 \%$ to $80 \%$ of the predicted value at baseline. Due to the low mortality rate caused by respiratory failure in our study $(n=4)$, we used the surrogate endpoints: progression of ILD and end-stage of ILD. Progression of ILD was defined as $\geq 10 \%$ relative decline in FVC or $\geq 5 \%$ to $<10 \%$ relative decline in FVC and $\geq 15 \%$ relative decline 
in DLco $(30,31)$. End-stage of ILD was defined as any of the following: a decline of FVC $<60 \%$, chronic respiratory failure requiring continuous oxygen supplementation, or death, as previously described with modifications $(32,33)$. Progression free survival, defined as the time to end-stage of ILD, was also evaluated.

The study was conducted in accordance with the Declaration of Helsinki (as revised in 2013). It was approved by the Ethics Committee of Kanazawa University Hospital (IRB protocol \#3028), and individual consent for this retrospective analysis was waived.

\section{$F B A L$}

FBAL was performed using a fiberoptic bronchoscope (Olympus) with a $4.9 \mathrm{~mm}$ outer diameter. Patients were sedated with midazolam and local anesthesia was achieved via inhalation of $1 \%$ lidocaine solution. The bronchoscope was introduced into the airway and wedged in the target segments. $50 \mathrm{~mL}$ of normal saline was instilled and immediately withdrawn by gentle manual suction. The procedure was repeated three times while remaining wedged in the targeted airway. We designated the first aliquot of the recovered BAL fluid as FBAL-1, the second as FBAL2, and the third as FBAL-3. Cases of BAL fluid recovery with less than $30 \%$ of instilled volume were excluded from the study. BAL was analyzed according to clinical practice guideline (34). In summary, BAL specimens were analyzed within one hour of their acquisition. Aliquots of BAL were sent to our diagnostic laboratories as well as microbiology and cytopathology. The remaining fluid was centrifuged at $280 \times \mathrm{g}$ for $1 \mathrm{~min}$ and then resuspended in $1 \mathrm{~mL}$ of phosphate-buffered saline. Total cell count was obtained by a hemocytometer and cell viability was determined by Trypan blue staining. BAL cells were then spun onto glass slides at $72.26 \times \mathrm{g}$ for $8 \mathrm{~min}$ using the Cytospin 3 (Thermo Shandon). The slides were then dried and stained with Giemsa stain. FBAL was performed by a pulmonologist using microscopy. The proportion of macrophages, lymphocytes, neutrophils, and eosinophils per 300 cells counted was recorded for every aliquot. The estimated value of total FBAL cell fraction (FBAL-total) was calculated as (FBAL-1 cell number $\times$ FBAL-1 cell fraction ratio + FBAL2 cell number $\times$ FBAL-2 cell fraction ratio + FBAL-3 cell number $\times$ FBAL- 3 cell fraction ratio)/(number of FBAL-1 cells + number of FBAL-2 cells + number of FBAL-3 cells), according to a previous study (35).

\section{Statistical analyses}

Continuous values were represented as the median and range. Unpaired comparisons of continuous variables were performed using the Kruskal-Wallis test or Mann-Whitney $\mathrm{U}$ test as appropriate. Categorical variables were compared using the chi-square test. Spearman's correlation coefficient was obtained for correlations. The Cox proportional hazard model was used to compare disease progression to endstage ILD. The results were shown as a hazard ratio (HR) with a $95 \%$ confidence interval (CI). Survival was analyzed using the Kaplan-Meier method and significance was determined with the log-rank test. All the statistical analyses were performed using EZR (36). A P value less than 0.05 was considered statistically significant.

\section{Results}

\section{Patient characteristics}

Of 79 SSc-ILD patients who underwent FBAL from January 2005 to December 2017, 11 patients were excluded; 1 patient had chronic respiratory failure at the time of FBAL, 1 patient had received FBAL for the diagnosis of bacterial pneumonia, 9 patients were excluded because the total volume of retrieved BAL fluid was under $30 \%$ of the instilled volume, as is suggested in ATS guidelines (34). The remaining 68 patients were included in this study (Figure S1). The sample size was determined by the number of cases during the study period. There are no missing data in this study.

The patient characteristics are summarized in Table 1. The median age was 56 years and $76.5 \%$ of patients were female. Three patients $(4.4 \%)$ were current smokers, 22 patients $(32.4 \%)$ were former smokers, and 43 patients $(63.2 \%)$ were never smokers. Eighteen patients (26.4\%) were taking oral corticosteroids (median dose of prednisolone, $8 \mathrm{mg}$ ), 1 patient was taking prednisolone and cyclosporine, and 1 patient was taking methotrexate at the time of BAL procedure. All patients had auto-antibodies including anti-topoisomerase I ( $\mathrm{n}=38)$, anti-U1RNP $(\mathrm{n}=9)$, anti-centromere $(n=9)$, anti-RNA polymerase $(n=9)$, or others $(\mathrm{n}=10)$. Most HRCTs demonstrated a non-specific interstitial pneumonia (NSIP) pattern with peripheral and basilar predominant ground-glass opacities and reticulation without significant honeycombing on chest HRCT scan. The median FVC\% predicted, forced expiratory volumeone second $\left(\mathrm{FEV}_{1}\right) \%$ predicted, and DLco\% predicted were 
Table 1 Patient characteristics at baseline

\begin{tabular}{|c|c|}
\hline Parameter & Value \\
\hline Patients, $\mathrm{n}$ & 68 \\
\hline Age, years [median (range)] & 56 [46-63] \\
\hline Gender female [n (\%)] & $52(76.5)$ \\
\hline Smoking status, Current/Former/Never, n & $3 / 22 / 43$ \\
\hline Steroid/Immunosuppressant use [n (\%)] & $20(29.4)$ \\
\hline Diffuse cutaneous SSc [n (\%)] & $54(79.4)$ \\
\hline mRSS [median (range)] & $11[4-22]$ \\
\hline \multicolumn{2}{|l|}{ Auto-antibodies } \\
\hline Anti-Scl-70 [n (\%)] & $38(55.9)$ \\
\hline Anti-U1RNP [n (\%)] & $9(13.2)$ \\
\hline Anti-Centromere [n (\%)] & $9(13.2)$ \\
\hline Anti-RNA polymerase [n (\%)] & $9(13.2)$ \\
\hline Others [n (\%)] & $10(14.7)$ \\
\hline \multicolumn{2}{|l|}{ Chest HRCT findings } \\
\hline UIP/NSIP pattern, $n / n$ & $1 / 67$ \\
\hline \multicolumn{2}{|l|}{ Distribution } \\
\hline Bronchovascular/Subpleural/Diffuse, $n / n / n$ & $2 / 61 / 5$ \\
\hline \multicolumn{2}{|l|}{ Characteristics } \\
\hline Ground-glass opacities [n (\%)] & $35(51.5)$ \\
\hline Traction bronchiectasis [n (\%)] & $27(39.7)$ \\
\hline Reticular abnormalities [n (\%)] & $40(58.8)$ \\
\hline Honeycombing [n (\%)] & $4(5.9)$ \\
\hline \multicolumn{2}{|l|}{ Pulmonary function tests } \\
\hline FVC, \% predicted [median (range)] & 88.8 (82.0-99.0) \\
\hline FEV1, \% predicted [median (range)] & 89.8 (79.9-100.4) \\
\hline DLco, \% predicted [median (range)] & $58.2(46.0-68.7)$ \\
\hline
\end{tabular}

mRSS, the modified Rodnan skin score; HRCT, high-resolution computed tomography; UIP, usual interstitial pneumonia; NSIP, non-specific interstitial pneumonia; FVC, forced vital capacity; $\mathrm{FEV}_{1}$, forced expiratory volume-one second; DLco, a diffusion lung capacity for carbon monoxide.

$88.8 \%, 89.8 \%$, and $58.2 \%$, respectively.

\section{Analysis of FBAL}

The median percentage of fluid recovered was $47 \%$ (range, $40-53 \%)$. FBAL was performed in the middle lobe in 38 patients $(55.9 \%)$ had and in the lower lobe in 30 patients (44.1\%). Cell fractionation of BAL showed that the median percentages of macrophages, lymphocytes, neutrophils, and eosinophils were $79.0 \%, 8.0 \%, 7.3 \%, 1.7 \%$ in FBAL$1,82.4 \%, 11.0 \%, 3.0 \%, 0.7 \%$ in $\mathrm{FBAL}-2,83.0 \%, 12.5 \%$, $2.3 \%, 0.5 \%$ in FBAL- 3 , and $81.7 \%, 11.6 \%, 3.5 \%, 0.8 \%$ in the estimated total FBAL (FBAL-total), respectively (Table 2). The percentage of neutrophils and eosinophils was significantly lower in FBAL-3 compared to FBAL-1. The percentages of macrophages and lymphocytes were higher in FBAL-3 compared to FBAL-1, but it was not statistically significant. Although there were more ciliated/epithelial cells in FBAL-1 than in FBAL-2 or FBAL-3, the exact number was not assessed in this study.

We next examined whether or not the treatment regimen, autoantibodies, or smoking status affected the proportions of cells obtained from the various fractions. Steroid/immunosuppressant use reduced the number of eosinophils in FBAL-3 and FBAL-total. However, it did not affect the other cell types, including neutrophils (Table S1). The patients with anti-Scl-70 autoantibodies had more neutrophils in FBAL-3 than those without antiScl-70 autoantibodies (Table S2). In contrast, there were no marked differences in the proportions of cells between smokers and non-smokers (Table S3).

The correlation between FBAL levels and pulmonary function variables at baseline are shown in Table 3. Both lymphocytes in FBAL-3 and FBAL-total significantly correlated to $\mathrm{FVC} \%$ predicted $(\mathrm{r}=0.348$ and $\mathrm{r}=0.296$, respectively) and DLco\% predicted ( $\mathrm{r}=0.269$ and $\mathrm{r}=0.261$, respectively). Neutrophils in FBAL-2, -3, and FBALtotal had a significant inverse correlation with $\mathrm{FVC} \%$ predicted $(r=-0.420, r=-0.362$ and $r=-0.409$, respectively). Macrophages and eosinophils showed no correlation with pulmonary function variables.

\section{Prognostic value of FBAL}

Of the 68 patients, 2 patients were excluded for further analysis because their DLco was less than $30 \%$ of the predicted value at baseline. Of the remaining 66 patients, $46(70 \%)$ developed progression of their ILD between 6 to 147 months after entering the study (median 88 months). FBAL findings were not predictive of the progression of ILD in univariate analysis. 19 patients (29\%) progressed to end-stage ILD; 13 patients had a decline of FVC $<60 \%$ and 6 patients developed chronic respiratory failure. Of the 19 patients, 4 patients died during the study period. In the 
Table 2 Cellular profile of FBAL in SSc-ILD

\begin{tabular}{lcccc}
\hline & Macrophages, \% & Lymphocytes, \% & Neutrophils, \% & Eosinophils, \% \\
\hline FBAL-1 & $79.0(64.5-86.9)$ & $8.0(4.7-17.2)$ & $7.3(3.7-14.2)$ & $1.7(0.3-3.3)$ \\
FBAL-2 & $82.4(73.8-89.0)$ & $11.0(7.3-17.3)$ & $3.0(1.7-5.4)^{\star}$ & $0.7(0.0-2.0)$ \\
FBAL-3 & $83.0(74.0-90.4)$ & $12.5(6.2-19.8)$ & $2.3(1.3-4.4)^{\star}$ & $0.5(0.0-2.0)^{\#}$ \\
FBAL-total & $81.7(72.8-89.1)$ & $11.6(6.7-17.0)$ & $3.5(1.9-6.2)$ & $0.8(0.3-2.0)$ \\
\hline
\end{tabular}

FBAL, fractional analysis of bronchoalveolar lavage. ${ }^{*} \mathrm{P}<0.05$ as compared with neutrophils in FBAL-1. "P $<0.05$ as compared with eosinophils in FBAL-1.

Table 3 Correlation between FBAL and pulmonary function variables

\begin{tabular}{|c|c|c|c|c|}
\hline & \multicolumn{2}{|c|}{ FVC (\%pred) } & \multicolumn{2}{|c|}{ DLco (\%pred) } \\
\hline \multicolumn{5}{|l|}{ Macrophages } \\
\hline FBAL-1 & 0.082 & 0.507 & -0.080 & 0.521 \\
\hline FBAL-2 & -0.047 & 0.705 & -0.170 & 0.165 \\
\hline FBAL-total & -0.120 & 0.332 & -0.214 & 0.082 \\
\hline \multicolumn{5}{|l|}{ Lymphocytes } \\
\hline FBAL-1 & 0.111 & 0.372 & 0.135 & 0.276 \\
\hline FBAL-2 & 0.226 & 0.064 & 0.247 & 0.042 \\
\hline \multicolumn{5}{|l|}{ Neutrophils } \\
\hline FBAL-1 & -0.218 & 0.076 & -0.051 & 0.681 \\
\hline FBAL-2 & -0.420 & $<0.001$ & -0.214 & 0.079 \\
\hline FBAL-3 & -0.362 & 0.002 & -0.140 & 0.256 \\
\hline FBAL-total & -0.409 & $<0.001$ & -0.212 & 0.083 \\
\hline \multicolumn{5}{|l|}{ Eosinophils } \\
\hline FBAL-1 & -0.046 & 0.712 & 0.093 & 0.456 \\
\hline FBAL-2 & 0.010 & 0.934 & 0.101 & 0.414 \\
\hline
\end{tabular}

FBAL, fractional analysis of bronchoalveolar lavage; FVC, forced vital capacity; DLco, a diffusion lung capacity for carbon monoxide.

univariate analysis, the presence of neutrophilia on FBAL3 was associated with end-stage ILD (HR 1.093, 95\% CI: $1.003-1.190, \mathrm{P}=0.043$ ) (Table 4). There were no statistically significant associations between cellular profile in FBALtotal and progression of ILD or end-stage ILD.
A receiver operating characteristic (ROC) curve analysis was used to determine the optimal cut-off levels of neutrophils in FBAL-3 for predicting risk of the end-stage ILD. The optimal cut-off level was 3\% for neutrophils in FBAL-3 (sensitivity 63.2\%, specificity 68.1\%). Kaplan- 
Table 4 Associated factors of FBAL for the progression of ILD and end-stages of ILD

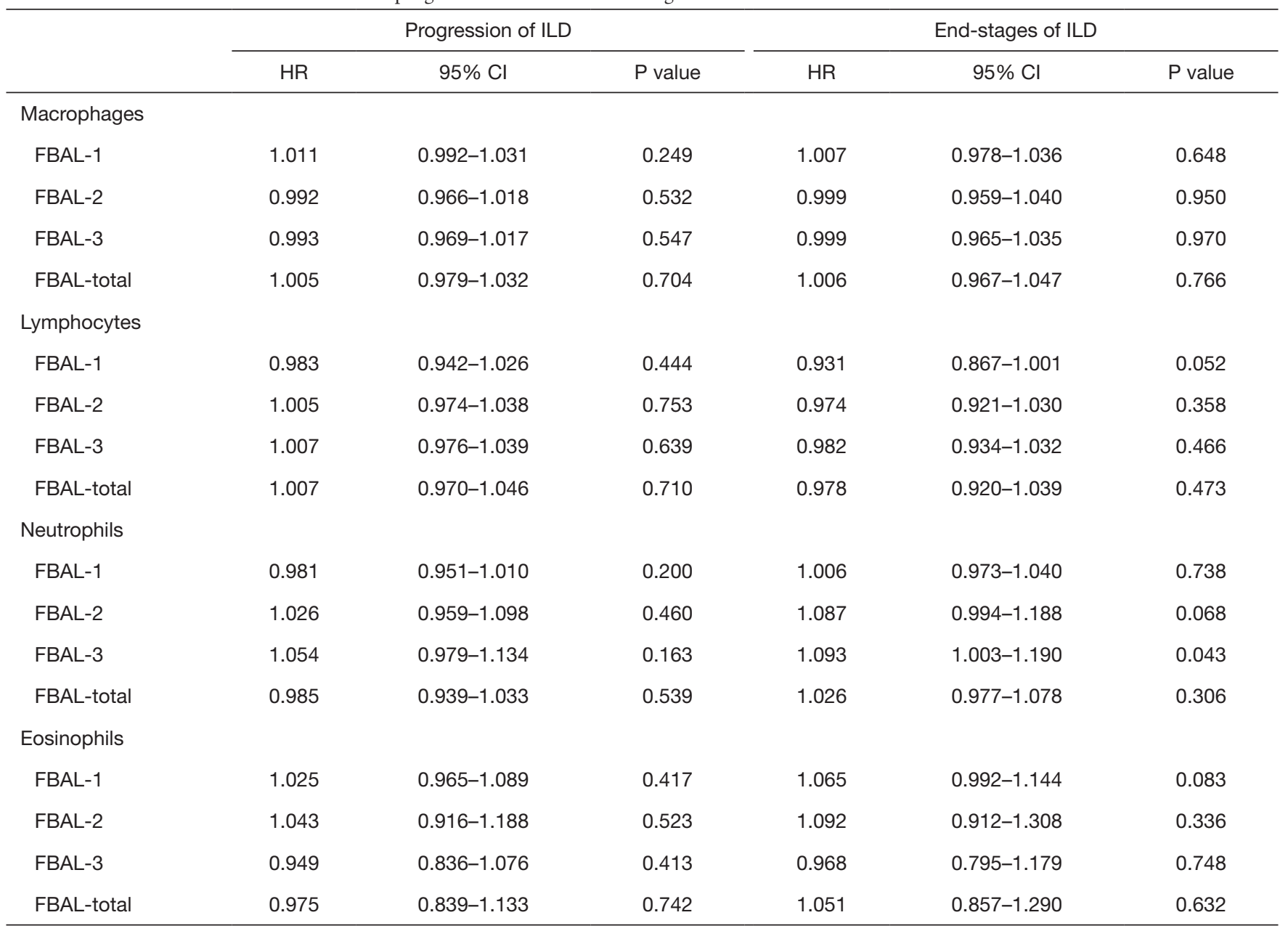

FBAL, fractional analysis of bronchoalveolar lavage.

Meier analysis showed that a neutrophil level $\geq 3 \%$ in FBAL-3 was associated with worse outcomes compared to neutrophils $<3 \%$ (log-rank test, $\mathrm{P}=0.025$ ) (Figure 1). The recovery rate of FBAL was similar between the groups (median $45 \%$ vs. $47 \%, \mathrm{P}=0.955$ ).

\section{Discussion}

We found that FBAL is a useful tool for predicting the risk of end-stage SSc-ILD. Although the FBAL-total (pooled BAL) did not predict progression and end-stage of SScILD, an increased percentage of neutrophils in FBAL3 was associated with development end-stage SSc-ILD. In addition, neutrophils $\geq 3 \%$ in FBAL-3 was a reliable predictor of the outcome. To the best of our knowledge, this is the first study evaluating the utility of FBAL in SSc-
ILD.

There are many studies investigating the cellular composition of BAL fluid in patients with SSc-ILD. Most studies defined an increased percentage of granulocytes including neutrophils (higher than $3 \%$ to $4 \%$ ), eosinophils (higher than $2 \%$ to $2.5 \%$ ), and/or lymphocytes (higher than $15 \%$ ) as "alveolitis" (37). Alveolitis is associated with more severe ILD but poorly predicts functional disease progression and mortality in SSc-ILD (37). This data supports our finding that an increased percentage of neutrophils in FBAL-total (pooled BAL) was inversely correlated with $\mathrm{FVC} \%$ predicted but did not predict disease progression or end-stage ILD. Although several factors, such as a smoking habit, have been reported to affect the cellular composition of BAL (38), the treatment regimens, autoantibodies, and smoking status did not affect the 


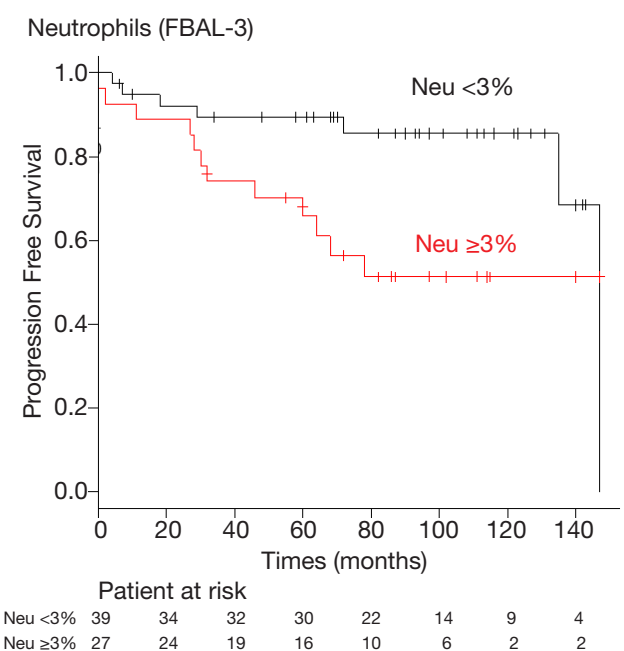

Figure 1 Comparison of progression free survival according to neutrophil levels in FBAL-3. FBAL, fractional bronchoalveolar lavage.

proportions of cells obtained from the various fractions in our cohort.

FBAL is a simple procedure for sampling cellular elements of the lung that does not require additional new techniques. FBAL provides more precise information on immune cell populations at the levels of the small airway and alveolar compartments. Neutrophils and eosinophils predominantly localize in bronchiolar space (FBAL-1), while alveolar macrophages and lymphocytes mainly localize in alveolar space (FBAL-2, -3) (27,39). These patterns are in agreement with the patterns of FBAL in SSc-ILD from our study. A pooled BAL fluid contains both bronchiolar and alveolar compartments, and inflammation in either anatomical compartment can lead to changes in the pooled BAL fluid. For example, the proportion of neutrophils in pooled BAL fluid may be highly affected by the degree of neutrophils at the airway level. In the present study, neutrophils in FBAL-2 and FBAL-3 (alveolar space) had a significant inverse correlation with the $\mathrm{FVC} \%$ predicted, but neutrophils in FBAL-1 (bronchiolar space) did not (Table 3). Thus, FBAL-2 and FBAL-3 may reflect the alveolar inflammation and severity of ILD more precisely compared to FBAL-1.

Our study demonstrated that a higher percentage of neutrophils in FBAL-3 is associated with the development of end-stage SSc-ILD. It is likely that it did not predict intermediate disease progression because the course of pulmonary function is thought to be highly variable in SSc-
ILD (40). The pathogenesis of SSc-ILD is characterized by recurrent and chronic endothelial injury which promotes the recruitment of inflammatory cells and the production of profibrotic mediators (26). Neutrophils, as inflammatory mediators, likely have prognostic value as markers of ongoing endothelial injury. Several studies have shown that activated neutrophils have the potential to release agents, including reactive oxygen species and proteases, capable of direct endothelial injury and alteration of cytokine signaling (41-44). Recent studies have shown that microparticles released from activated platelets in the blood of SSc patients are abundant and express a damage-associated molecular pattern called high-mobility-group-box-1 (HMGB1) $(45,46)$. HMGB1 activates neutrophil extracellular traps (NETs), promoting fibrogenesis after endothelial cell damage and favoring tissue remodeling (47). NETs are involved in not only inflammation but also promoting collagen fiber proliferation and IL-17-mediated fibrosis (48). Thus, neutrophils may play pro-inflammatory and profibrotic roles in SSc-ILD.

Several limitations associated with the present study warrant mention. First, this was a retrospective, single center study with a small number of enrolled patients. A large, multicenter, prospective studies would be needed to validate our findings. Second, we performed BAL in a $3 \times 50 \mathrm{~mL}$ protocol. While this is generally considered standard practice for FBAL, significant institutionspecific differences exist in technical procedures and in the processing of BAL fluid $(34,49)$. A technical standard needs to be established for the use of FBAL protocols for our results to be put into clinical practice. Finally, the mechanisms underlying the association between FBAL findings and disease severity/outcome remain unclear. Further studies will be needed to examine the cytokine levels under conditions of endothelial injury and profibrotic markers in the different fractions of FBAL.

In conclusion, we demonstrated that an elevated percentage of neutrophils in FBAL-3 was associated with the risk of the end-stage of SSc-ILD. We believe FBAL captures the disease state associated with mortality better than pooled BAL and may aid clinical decisions for therapeutic interventions and follow-up.

\section{Acknowledgments}

We thank all the patients who participated in our clinical study and medical staff who contributed to the treatment and care of the patients. We thank all the dermatologists 
and rheumatologists (Kanazawa University Hospital) for the diagnosis of SSc. We also thank Y Tanaka (Prevention medicine, Northwestern University) for giving us advice on the statistical analysis and Lango Sichizya and Michael Alexander (Pulmonary and Critical Care, Northwestern University) for comments that greatly improved the manuscript.

Funding: None.

\section{Footnote}

Reporting Checklist: The authors have completed the STROBE Checklist. Available at https://dx.doi. org/10.21037/jtd-20-2596

Data Sharing Statement: Available at https://dx.doi. org/10.21037/jtd-20-2596

Peer Review File: Available at https://dx.doi.org/10.21037/ jtd-20-2596

Conflicts of Interest: All authors have completed the ICMJE uniform disclosure form (available at https://dx.doi. org/10.21037/jtd-20-2596). The authors have no conflicts of interest to declare.

Ethical Statement: The authors are accountable for all aspects of the work in ensuring that questions related to the accuracy or integrity of any part of the work are appropriately investigated and resolved. The study was conducted in accordance with the Declaration of Helsinki (as revised in 2013). The study was approved by the Ethics Committee of Kanazawa University Hospital (IRB protocol \#3028) and individual consent for this retrospective analysis was waived.

Open Access Statement: This is an Open Access article distributed in accordance with the Creative Commons Attribution-NonCommercial-NoDerivs 4.0 International License (CC BY-NC-ND 4.0), which permits the noncommercial replication and distribution of the article with the strict proviso that no changes or edits are made and the original work is properly cited (including links to both the formal publication through the relevant DOI and the license). See: https://creativecommons.org/licenses/by-nc-nd/4.0/.

\section{References}

1. Denton CP, Khanna D. Systemic sclerosis. Lancet
2017;390:1685-99.

2. Al-Dhaher FF, Pope JE, Ouimet JM. Determinants of morbidity and mortality of systemic sclerosis in Canada. Semin Arthritis Rheum 2010;39:269-77.

3. Nihtyanova SI, Schreiber BE, Ong VH, et al. Prediction of pulmonary complications and long-term survival in systemic sclerosis. Arthritis Rheumatol 2014;66:1625-35.

4. Hoffmann-Vold AM, Aalokken TM, Lund MB, et al. Predictive value of serial high-resolution computed tomography analyses and concurrent lung function tests in systemic sclerosis. Arthritis Rheumatol 2015;67:2205-12.

5. Goh NS, Hoyles RK, Denton CP, et al. Short-Term Pulmonary Function Trends Are Predictive of Mortality in Interstitial Lung Disease Associated With Systemic Sclerosis. Arthritis Rheumatol 2017;69:1670-8.

6. Hoffmann-Vold AM, Fretheim H, Halse AK, et al. Tracking Impact of Interstitial Lung Disease in Systemic Sclerosis in a Complete Nationwide Cohort. Am J Respir Crit Care Med 2019;200:1258-66.

7. Suliman YA, Dobrota R, Huscher D, et al. Brief Report: Pulmonary Function Tests: High Rate of FalseNegative Results in the Early Detection and Screening of Scleroderma-Related Interstitial Lung Disease. Arthritis Rheumatol 2015;67:3256-61.

8. Winstone TA, Assayag D, Wilcox PG, et al. Predictors of mortality and progression in scleroderma-associated interstitial lung disease: a systematic review. Chest 2014;146:422-36.

9. Phan SH. The myofibroblast in pulmonary fibrosis. Chest 2002;122:286S-9S.

10. Distler O, Highland KB, Gahlemann M, et al. Nintedanib for Systemic Sclerosis-Associated Interstitial Lung Disease. N Engl J Med 2019;380:2518-28.

11. Sullivan KM, Goldmuntz EA, Keyes-Elstein L, et al. Myeloablative Autologous Stem-Cell Transplantation for Severe Scleroderma. N Engl J Med 2018;378:35-47.

12. Distler O, Volkmann ER, Hoffmann-Vold AM, et al. Current and future perspectives on management of systemic sclerosis-associated interstitial lung disease. Expert Rev Clin Immunol 2019;15:1009-17.

13. Davidson KR, Ha DM, Schwarz MI, et al. Bronchoalveolar lavage as a diagnostic procedure: a review of known cellular and molecular findings in various lung diseases. J Thorac Dis 2020;12:4991-5019.

14. Raghu G, Remy-Jardin M, Myers JL, et al. Diagnosis of Idiopathic Pulmonary Fibrosis. An Official ATS/ERS/JRS/ ALAT Clinical Practice Guideline. Am J Respir Crit Care Med 2018;198:e44-e68. 
15. Raghu G, Remy-Jardin M, Ryerson CJ, et al. Diagnosis of Hypersensitivity Pneumonitis in Adults. An Official ATS/ JRS/ALAT Clinical Practice Guideline. Am J Respir Crit Care Med 2020;202:e36-e69.

16. Frye BC, Schupp JC, Rothe ME, et al. The value of bronchoalveolar lavage for discrimination between healthy and diseased individuals. J Intern Med 2020;287:54-65.

17. Friedmann D, Unger S, Keller B, et al. Bronchoalveolar Lavage Fluid Reflects a T(H)1-CD21(low) B-Cell Interaction in CVID-Related Interstitial Lung Disease. Front Immunol 2021;11:616832.

18. Frigieri L, Mormile F, Grilli N, et al. Bilateral bronchoalveolar lavage in progressive systemic sclerosis: interlobar variability, lymphocyte subpopulations, and functional correlations. Respiration 1991;58:132-40.

19. Behr J, Vogelmeier C, Beinert T, et al. Bronchoalveolar lavage for evaluation and management of scleroderma disease of the lung. Am J Respir Crit Care Med 1996;154:400-6.

20. White B, Moore WC, Wigley FM, et al. Cyclophosphamide is associated with pulmonary function and survival benefit in patients with scleroderma and alveolitis. Ann Intern Med 2000;132:947-54.

21. De Santis M, Bosello S, La Torre G, et al. Functional, radiological and biological markers of alveolitis and infections of the lower respiratory tract in patients with systemic sclerosis. Respir Res 2005;6:96.

22. Strange C, Bolster MB, Roth MD, et al. Bronchoalveolar lavage and response to cyclophosphamide in scleroderma interstitial lung disease. Am J Respir Crit Care Med 2008;177:91-8.

23. Witt C, Borges AC, John M, et al. Pulmonary involvement in diffuse cutaneous systemic sclerosis: broncheoalveolar fluid granulocytosis predicts progression of fibrosing alveolitis. Ann Rheum Dis 1999;58:635-40.

24. Kowal-Bielecka O, Kowal K, Rojewska J, et al. Cyclophosphamide reduces neutrophilic alveolitis in patients with scleroderma lung disease: a retrospective analysis of serial bronchoalveolar lavage investigations. Ann Rheum Dis 2005;64:1343-6.

25. Goh NS, Veeraraghavan S, Desai SR, et al. Bronchoalveolar lavage cellular profiles in patients with systemic sclerosisassociated interstitial lung disease are not predictive of disease progression. Arthritis Rheum 2007;56:2005-12.

26. Solomon JJ, Olson AL, Fischer A, et al. Scleroderma lung disease. Eur Respir Rev 2013;22:6-19.

27. Taniuchi N, Ghazizadeh M, Enomoto T, et al. Evaluation of fractional analysis of bronchoalveolar lavage combined with cellular morphological features. Int J Med Sci 2009;6:1-8.

28. Ratjen F, Rietschel E, Griese M, et al. Fractional analysis of bronchoalveolar lavage fluid cytology in cystic fibrosis patients with normal lung function. Bronchoalveolar lavage for the evaluation of anti-inflammatory treatment (BEAT) study group. Eur Respir J 2000;15:141-5.

29. van den Hoogen F, Khanna D, Fransen J, et al. 2013 classification criteria for systemic sclerosis: an American college of rheumatology/European league against rheumatism collaborative initiative. Ann Rheum Dis 2013;72:1747-55.

30. Khanna D, Mittoo S, Aggarwal R, et al. Connective Tissue Disease-associated Interstitial Lung Diseases (CTD-ILD)Report from OMERACT CTD-ILD Working Group. J Rheumatol 2015;42:2168-71.

31. Flaherty KR, Wells AU, Cottin V, et al. Nintedanib in Progressive Fibrosing Interstitial Lung Diseases. N Engl J Med 2019;381:1718-27.

32. Medsger TA, Jr., Silman AJ, Steen VD, et al. A disease severity scale for systemic sclerosis: development and testing. J Rheumatol 1999;26:2159-67.

33. Hoshino K, Satoh T, Kawaguchi Y, et al. Association of hepatocyte growth factor promoter polymorphism with severity of interstitial lung disease in Japanese patients with systemic sclerosis. Arthritis Rheum 2011;63:2465-72.

34. Meyer KC, Raghu G, Baughman RP, et al. An official American Thoracic Society clinical practice guideline: the clinical utility of bronchoalveolar lavage cellular analysis in interstitial lung disease. Am J Respir Crit Care Med 2012;185:1004-14.

35. Matsuda K, Satoh M, Kawanami O. Airway and alveolar inflammation assessments with bronchoalveolar lavage in various interstitial lung disorders. Nihon Kyobu Shikkan Gakkai Zasshi 1992;30:827-34.

36. Kanda Y. Investigation of the freely available easy-touse software 'EZR' for medical statistics. Bone Marrow Transplant 2013;48:452-8.

37. Kowal-Bielecka O, Kowal K, Highland KB, et al. Bronchoalveolar lavage fluid in scleroderma interstitial lung disease: technical aspects and clinical correlations: review of the literature. Semin Arthritis Rheum 2010;40:73-88.

38. Bargagli E, Cameli P, Carleo A, et al. The effect of cigarette smoking on bronchoalveolar lavage protein profiles from patients with different interstitial lung diseases. Panminerva Med 2020;62:109-15.

39. Hartl D, Tirouvanziam R, Laval J, et al. Innate Immunity 
of the Lung: From Basic Mechanisms to Translational Medicine. J Innate Immun 2018;10:487-501.

40. Adamson IY, Bowden DH. Response of mouse lung to crocidolite asbestos. 2. Pulmonary fibrosis after long fibres. J Pathol 1987;152:109-17.

41. Barnes TC, Spiller DG, Anderson ME, et al. Endothelial activation and apoptosis mediated by neutrophildependent interleukin 6 trans-signalling: a novel target for systemic sclerosis? Ann Rheum Dis 2011;70:366-72.

42. Maslen CL, Hall ND, Woolf AD, et al. Enhanced oxidative metabolism of neutrophils from patients with systemic sclerosis. Br J Rheumatol 1987;26:113-7.

43. Casciola-Rosen L, Wigley F, Rosen A. Scleroderma autoantigens are uniquely fragmented by metal-catalyzed oxidation reactions: implications for pathogenesis. J Exp Med 1997;185:71-9.

44. Barnes TC, Anderson ME, Edwards SW, et al. Neutrophilderived reactive oxygen species in SSc. Rheumatology (Oxford) 2012;51:1166-9.

Cite this article as: Kase K, Watanabe S, Saeki K, Waseda Y, Takato H, Ichikawa Y, Murata A, Yasui M, Noriyuki O, Hara J, Sone T, Abo M, Kimura H, Kasahara K. Fractional analysis of bronchoalveolar lavage in systemic sclerosis-associated interstitial lung disease. J Thorac Dis 2021;13(7):4146-4155. doi: $10.21037 /$ jtd-20-2596
45. Maugeri N, Capobianco A, Rovere-Querini P, et al. Platelet microparticles sustain autophagy-associated activation of neutrophils in systemic sclerosis. Sci Transl Med 2018;10:eaao3089.

46. Didier K, Giusti D, Le Jan S, et al. Neutrophil Extracellular Traps Generation Relates with Early Stage and Vascular Complications in Systemic Sclerosis. J Clin Med 2020;9:2136.

47. Hayakawa K, Pham LD, Katusic ZS, et al. Astrocytic highmobility group box 1 promotes endothelial progenitor cell-mediated neurovascular remodeling during stroke recovery. Proc Natl Acad Sci U S A 2012;109:7505-10.

48. Chrysanthopoulou A, Mitroulis I, Apostolidou E, et al. Neutrophil extracellular traps promote differentiation and function of fibroblasts. J Pathol 2014;233:294-307.

49. Haslam PL, Baughman RP. Report of ERS Task Force: guidelines for measurement of acellular components and standardization of BAL. Eur Respir J 1999;14:245-8. 


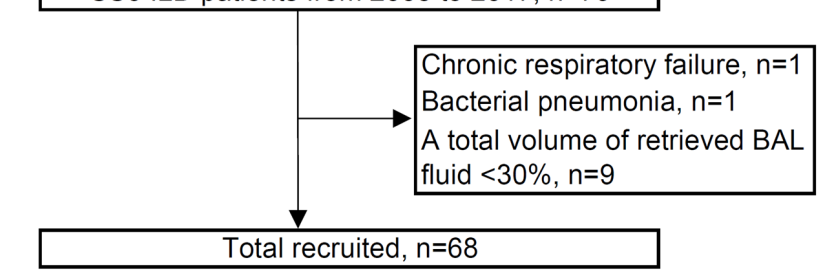

Figure S1 Consort diagram of SSc-ILD patients.

Table S1 Cellular profile of FBAL between subjects with and without steroid/immunosuppressant use

\begin{tabular}{|c|c|c|c|c|c|}
\hline \multirow{2}{*}{$\begin{array}{l}\text { Cellular profile } \\
\text { Macrophages }\end{array}$} & \multicolumn{2}{|c|}{ Steroid/Immuno-suppressant use (-) } & \multicolumn{2}{|c|}{ Steroid/Immuno-suppressant use (+) } & \multirow[t]{2}{*}{$P$ value } \\
\hline & & & & & \\
\hline FBAL-1 & 76.7 & (61.7-88.3) & 80.4 & (70.8-86.9) & 0.598 \\
\hline FBAL-2 & 82.7 & (75.0-88.0) & 81.4 & (68.7-89.8) & 0.893 \\
\hline FBAL-3 & 81.7 & $(74.0-88.7)$ & 88.0 & (84.2-93.1) & 0.291 \\
\hline FBAL-total & 81.3 & (73.9-88.2) & 84.4 & (70.8-91.0) & 0.784 \\
\hline \multicolumn{6}{|l|}{ Lymphocytes } \\
\hline FBAL-1 & 8.0 & $(4.3-17.7)$ & 7.8 & $(5.7-15.8)$ & 0.891 \\
\hline FBAL-2 & 11.0 & $(8.3-14.7)$ & 11.8 & $(5.5-25.4)$ & 0.772 \\
\hline FBAL-3 & 13.0 & $(6.7-19.7)$ & 8.7 & $(4.9-21.4)$ & 0.447 \\
\hline FBAL-total & 11.9 & $(7.8-16.7)$ & 10.0 & $(5.4-22.2)$ & 0.666 \\
\hline \multicolumn{6}{|l|}{ Neutrophils } \\
\hline FBAL-1 & 7.9 & $(3.7-14.5)$ & 5.9 & $(3.4-12.6)$ & 0.502 \\
\hline FBAL-2 & 3.0 & $(1.3-5.3)$ & 3.5 & $(2.0-5.8)$ & 0.462 \\
\hline FBAL-3 & 2.0 & $(1.0-4.7)$ & 2.7 & $(1.5-4.0)$ & 0.656 \\
\hline FBAL-total & 3.5 & $(1.8-6.3)$ & 3.6 & $(2.3-6.7)$ & 0.851 \\
\hline \multicolumn{6}{|l|}{ Eosinophils } \\
\hline FBAL-1 & 2.0 & $(0.5-3.7)$ & 0.7 & $(0.3-2.2)$ & 0.077 \\
\hline FBAL-2 & 1.0 & $(0.0-2.3)$ & 0.3 & $(0.0-1.3)$ & 0.110 \\
\hline FBAL-3 & 1.0 & $(0.0-2.0)$ & 0.2 & $(0.0-0.9)$ & 0.034 \\
\hline FBAL-total & 1.0 & $(0.4-3.1)$ & 0.3 & $(0.1-0.9)$ & 0.024 \\
\hline
\end{tabular}


Table S2 Cellular profile of FBAL between subjects with and without anti-Scl-70 antibody

\begin{tabular}{|c|c|c|c|c|c|}
\hline Cellular profile & \multicolumn{2}{|c|}{ Anti-Scl-70 (-) } & \multicolumn{2}{|c|}{ Anti-Scl-70 (+) } & $P$ value \\
\hline FBAL-1 & 82.0 & $(57.9-86.4)$ & 77.3 & (65.5-88.9) & 0.935 \\
\hline FBAL-2 & 85.7 & $(79.8-89.1)$ & 79.0 & (72.4-89.0) & 0.132 \\
\hline FBAL-3 & 84.4 & $(78.1-91.5)$ & 81.7 & (72.8-89.0) & 0.148 \\
\hline \multicolumn{6}{|l|}{ Lymphocytes } \\
\hline FBAL-1 & 7.7 & $(4.6-18.5)$ & 9.7 & $(5.0-17.2)$ & 0.767 \\
\hline FBAL-2 & 11.0 & $(5.2-15.3)$ & 11.0 & $(7.3-18.0)$ & 0.574 \\
\hline FBAL-3 & 10.3 & $(5.8-19.8)$ & 13.0 & $(6.8-20.4)$ & 0.370 \\
\hline FBAL-1 & 7.0 & $(3.7-18.0)$ & 7.3 & $(3.5-12.5)$ & 0.605 \\
\hline FBAL-2 & 2.2 & $(1.6-4.3)$ & 3.7 & $(1.9-6.2)$ & 0.104 \\
\hline FBAL-3 & 1.9 & $(0.9-3.0)$ & 3.3 & $(1.9-6.2)$ & 0.008 \\
\hline FBAL-total & 3.2 & $(1.7-6.2)$ & 3.7 & $(2.1-7.2)$ & 0.351 \\
\hline \multicolumn{6}{|l|}{ Eosinophils } \\
\hline FBAL-1 & 1.7 & $(0.3-3.4)$ & 1.0 & $(0.3-4.2)$ & 0.785 \\
\hline FBAL-2 & 0.9 & $(0.3-1.4)$ & 0.7 & $(0.0-3.3)$ & 0.975 \\
\hline FBAL-3 & 0.7 & $(0.0-1.7)$ & 0.3 & $(0.0-2.7)$ & 0.791 \\
\hline
\end{tabular}


Table S3 Cellular profile of FBAL between non-smokers and smokers

\begin{tabular}{|c|c|c|c|c|c|}
\hline $\begin{array}{l}\text { Cellular profile } \\
\text { Macrophages }\end{array}$ & \multicolumn{2}{|c|}{ Non-smokers } & \multicolumn{2}{|c|}{ Smokers } & $P$ value \\
\hline FBAL-1 & 77.7 & $(64.3-87.7)$ & 83.2 & $(65.8-87.7)$ & 0.552 \\
\hline FBAL-2 & 82.0 & $(72.7-88.7)$ & 83.9 & (75.6-90.8) & 0.558 \\
\hline FBAL-3 & 83.0 & $(74.0-89.7)$ & 83.0 & (73.5-92.2) & 0.980 \\
\hline \multicolumn{6}{|l|}{ Lymphocytes } \\
\hline FBAL-1 & 10.7 & $(5.0-18.3)$ & 6.7 & $(3.1-11.2)$ & 0.062 \\
\hline FBAL-2 & 11.3 & $(8.3-19.0)$ & 10.7 & $(5.0-13.2)$ & 0.257 \\
\hline FBAL-3 & 12.7 & $(8.3-19.9)$ & 9.9 & $(4.8-19.1)$ & 0.484 \\
\hline FBAL-1 & 7.0 & $(3.0-16.7)$ & 7.8 & $(5.1-13.2)$ & 0.614 \\
\hline FBAL-2 & 3.0 & $(1.7-5.3)$ & 3.0 & $(1.8-5.6)$ & 0.633 \\
\hline FBAL-3 & 2.3 & $(1.3-4.0)$ & 2.5 & $(1.0-4.9)$ & 0.579 \\
\hline FBAL-total & 3.5 & $(1.9-6.9)$ & 3.8 & $(1.7-6.1)$ & 0.760 \\
\hline \multicolumn{6}{|l|}{ Eosinophils } \\
\hline FBAL-1 & 1.0 & $(0.3-3.0)$ & 2.2 & $(0.6-3.9)$ & 0.147 \\
\hline FBAL-2 & 0.7 & $(0.0-1.6)$ & 1.0 & $(0.1-2.8)$ & 0.257 \\
\hline FBAL-3 & 0.3 & $(0.0-1.7)$ & 1.0 & $(0.0-3.5)$ & 0.124 \\
\hline
\end{tabular}

